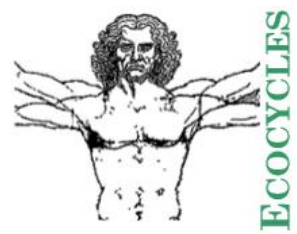

Ecocycles, Vol. 6, No. 2, pp. 49-53 (2020)

DOI: $\underline{10.19040 / \text { ecocycles.v6i2.159 }}$

CASE STUDY

\title{
Managing challenges of increasing complexity in sustainability
}

\author{
Anikó Klausmann-Dinya \\ Szent István University, Gödöllö / Gyöngyös, Hungary \\ E-mail address: dinya.aniko@supportivecoach.hu
}

\begin{abstract}
The paradigm under which our society and economy have operated until now has become obsolete in the 21st century. Some Nobel Prize-winner economists made it clear that we have to forget the paradigm currently dominating the global economy: "Let's privatize the benefits and socialize (distribute) its costs as much as possible!" The results of it are extremely and rapidly increasing inequalities, followed by unmanageable socio-economic - environmental tensions. It seems we could get out of this situation only with radical social and economic transformations, both globally and at the lower levels (countries, regions, localities). There isn't another solution just the social and economic paradigm exchange parallel with each other. Interconnected and not separated them. But many big challenges are coming up from the natural and the technological environment too and they are rooted in the human-made systems. So altogether we are facing the quickly changing complexity and an unknown situation in the history of humanity. We don't have appropriate methods and experiences how to deal with these new types of challenges but there isn't any other choice just to manage them. Despite this fact, there are very few studies about the causes and consequences of this rapidly growing problem in the era of growing sustainability risks. Similarly, there is very little practical information that provides actionable advice on how to manage these problems at different (global-, macro-, and micro-) levels of social and economic organizations. We define complexity as the number of components in a system plus the variety of relationships among these components plus the speed of changes of both the components and the relationships. Larger systems (like social - economic - ecological systems) are often very complex - but they may be more complicated if their behavior is unpredictable. Based on the global databases and reports we investigated the trials of countries how prepared they are for managing the growing complexity in the field of implementing the Sustainable Development Goals. We have found that in most of the countries (and globally too) experts are choosing the simplest way - they deal with the goals separately from each other and don't take into account the very complicated system of their interconnections and the feedback loops. We have tried to summarize some conclusions for the future about what would be the better approach to deal with the complexity.
\end{abstract}

Keywords - complexity challenges, complexity of sustainability

\section{WHY COMPLEXITY?}

This article is the first in a series to review issues related to the complexity of sustainability. As we make progress towards achieving the SDGs and analyze the experiences, it becomes increasingly clear, that the challenges that were initially less pronounced and that become increasingly important over time. One of these challenges is how well we can manage the complexity. This is the reason why we will only outline (expose) the essence of the issue this time and the details of its various dimensions later-on.
Starting with the implementation of SDG-s we have recognized the Earth as a closely linked human-environment system and provides a better understanding of the extent to which our shared progress as human beings is undermined by the ways in which we have gone about achieving it. Governments can lead the transformation of the world's social, economic, and environmental status towards universally beneficial outcomes when guided by the Sustainable Development Goals. But they must recognize that such transformation will involve tough choices and trade- 
offs. The new approach became widening that we have to bring the SDG-s together within one framework as an indivisible and universal whole. Therefore, not only the goals
The 2030 Agenda defined 17 Sustainable Development Goals and 169 targets. Tracking progress towards these targets at the global level is possible by indicators to be consistently

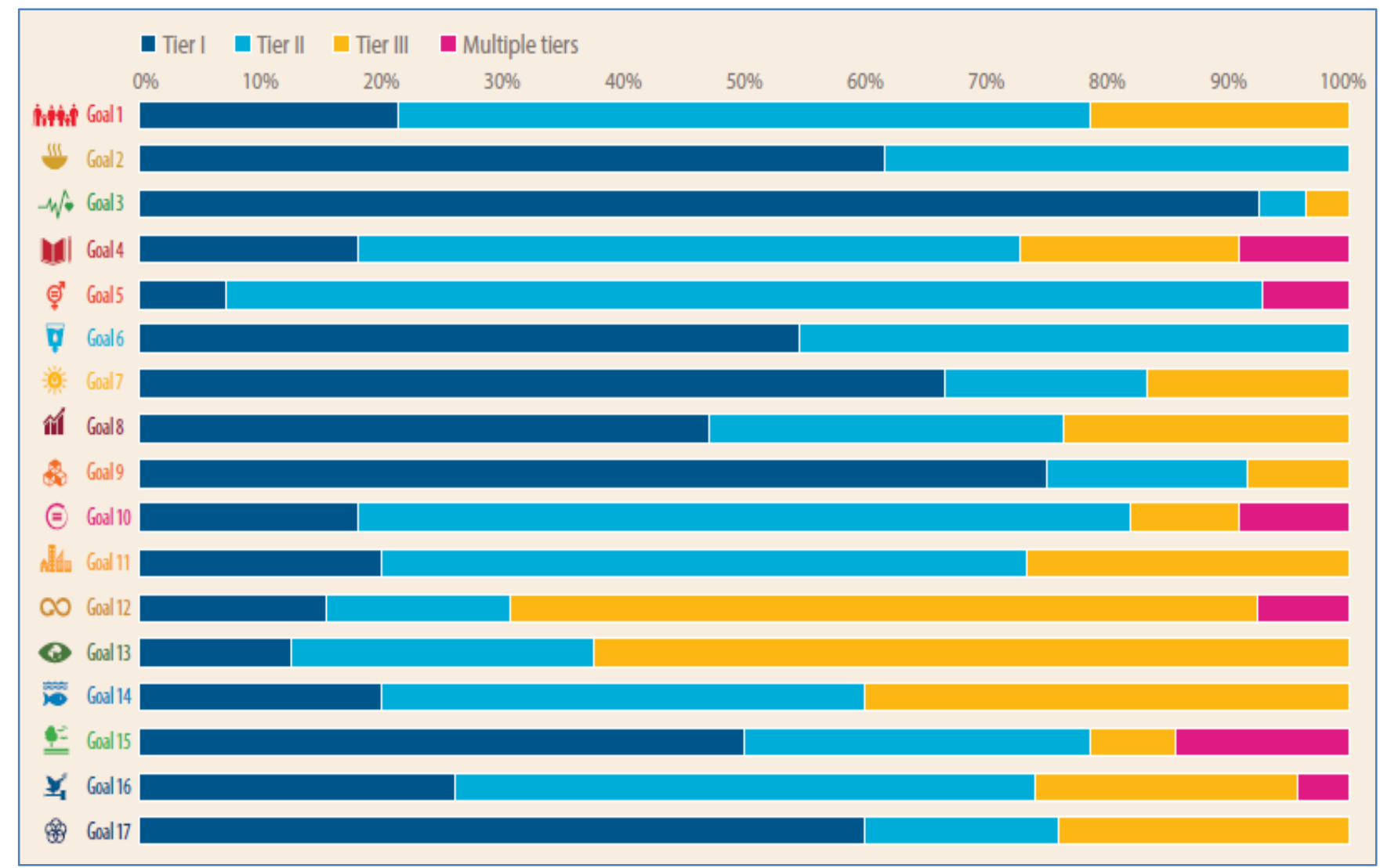

Figure 1. The share of indicators within each tier varies across goals (Source: United Nations, 2019a)

and targets but also the interactions among them, are brought into focus in the 2030 Agenda. The emphasis on interactions was likely influenced by the growing scientific understanding of the Earth, as a closely linked human-environment system. The Earth system is extremely complex and once beyond certain thresholds, even minor changes can lead to major events with drastic and irrevocable consequences. As a result, the Earth can reach tipping points. We need to solve the problems relating to poverty, inequality, and the rapidly deteriorating environment, and urgently. Indeed, the primary window of opportunity for change could be within the coming decade.

Every kind of solution must compete with powerful oppositional interests that benefit from the status quo or even intensify socially and environmentally damaging activities. The status quo may seem attractive in the short term, but it is clearly unsustainable and with negative longer-term consequences that will ultimately lead to chaotic and destructive outcomes.

\section{INTERACTIONS AMONG THE SUSTAINABLE DEVELOP- MENT GOALS - HOW TO MANAGE TO GORDIAN KNOT?}

defined and measured across countries. A team of international experts (the Inter-Agency and Expert Group on Sustainable Development Goal Indicators) has developed a global indicator framework that was agreed upon by the United Nations Statistical Commission in March 2017 and adopted by the General Assembly in July 2017. (Nilsson et al., 2017) There are currently 232 indicators in the global framework, classified into three tiers depending on their level of methodological development and the availability of data. Tier I indicators are well defined, with sufficient data regularly collected at the country level for reliable and timely global reporting; Tier II indicators are well defined, but data are not regularly collected at the country level; Tier III indicators are those for which definitions, methodologies or standards are under development.

As of May 2019, out of the 232 indicators, 104 were Tier I, 88 were Tier II, 34 are Tier III and 6 are categorized under multiple tiers. For example, more than 50 percent of the indicators for Goal 13 (climate action) are in Tier III. Currently available climate indicators are being used as proxies for monitoring the targets under Goal 13 (Figure 1). Another report (United Nations, 2019b) prepared by an independent group of scientists presents an objective assessment of where we are falling short and what needs to be done. The report highlights central entry points to leverage 
interlinkages and accelerate progress across all 17 Sustainable Development Goals.

Figure 2 shows the result of a systematic compilation of knowledge about causal interactions among the Sustainable Development Goals, extracted primarily at the target level and using the 7-point scale in terms of co-benefits and tradeoffs. In simple terms, a trade-off is when one indicator's achievement is increasing, and another must decrease (negative direction of interconnection). Co-benefit is the positive influence of each other. Based on the mapping of the summed scores of influencings (horizontal) and influenced (vertical) interactions among the Goals we could see the level of our present knowledge about the complex interconnected system. The Goals are given by their usual serial code numbers and symbols in the first column and the first row of the matrix. This assessment reveals the relative importance of the potential trade-offs, but the dominance of positive (blue) over negative (red) interactions in the current body of knowledge suggests that recent research has brought to the fore extensive co-benefits as well.

The figure also shows important blind spots or gaps in knowledge where certain cells in the matrix have been left blank. Of all possible target-level interactions, only about 10 percent were covered at least once. Aggregated to the Goals level, however, the matrix reveals that 92 percent of Goallevel interactions were assessed. In systemic terms, the figure

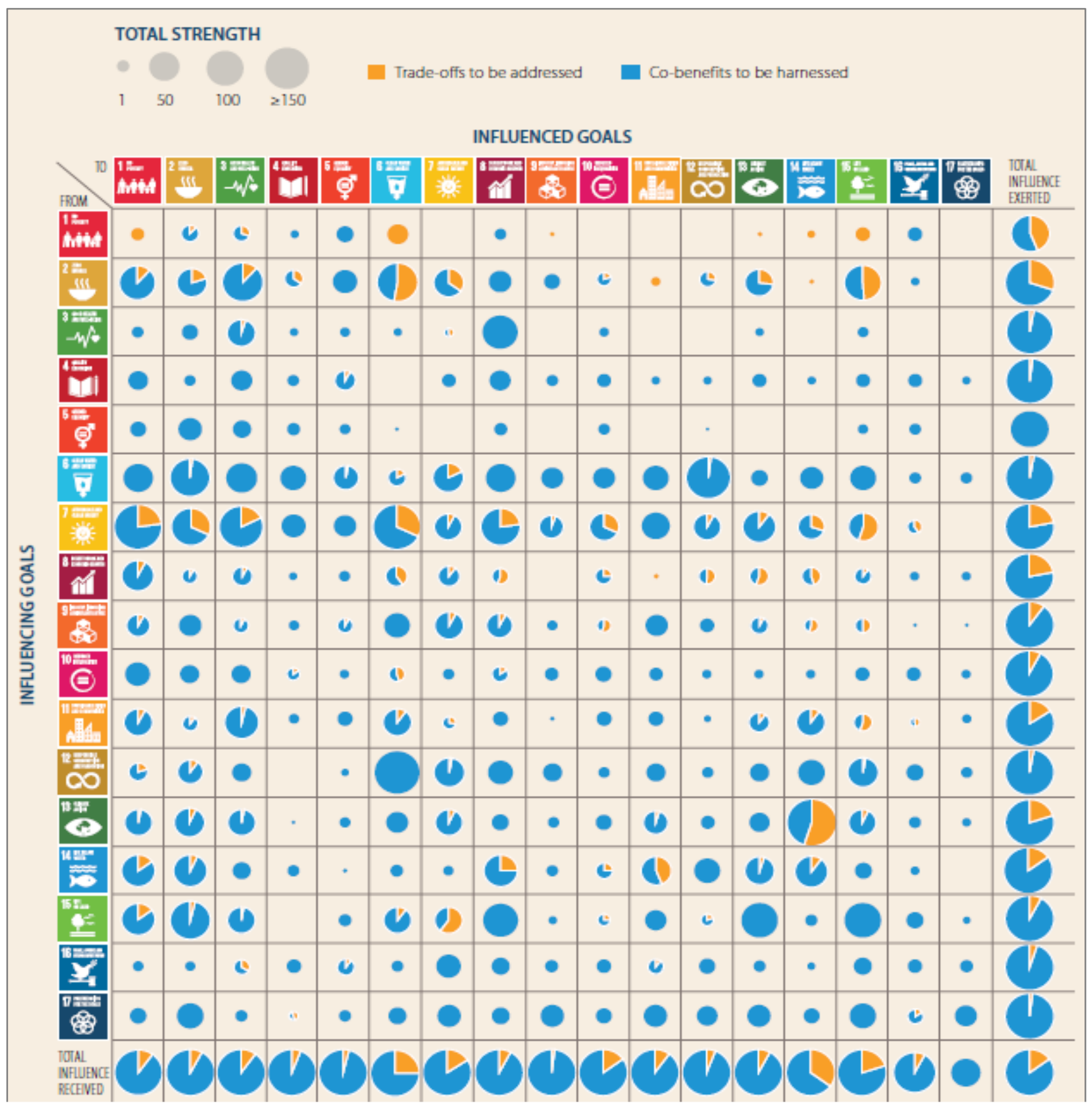

Figure 2. Interactions among Sustainable Development Goals (United Nations, 2019b) 
suggests that change towards achieving the Sustainable Development Goals offers many opportunities for reinforcing rather than inhibiting itself.

Concerning country-level assessments and forecasts, in 2019, one study found that no country was on track to meet all of the Goals by 2030. (Sachs et al., 2019) A recent report by Paris21 found that even highly developed countries are still not able to report more than $40-50 \%$ of the SDG indicators (Paris21, 2020).

Achieving more equitable and balanced development within the political space of the 2030 Agenda is possible only by engaging with the systems that connect people and nature to their guiding goals (Figure 3).

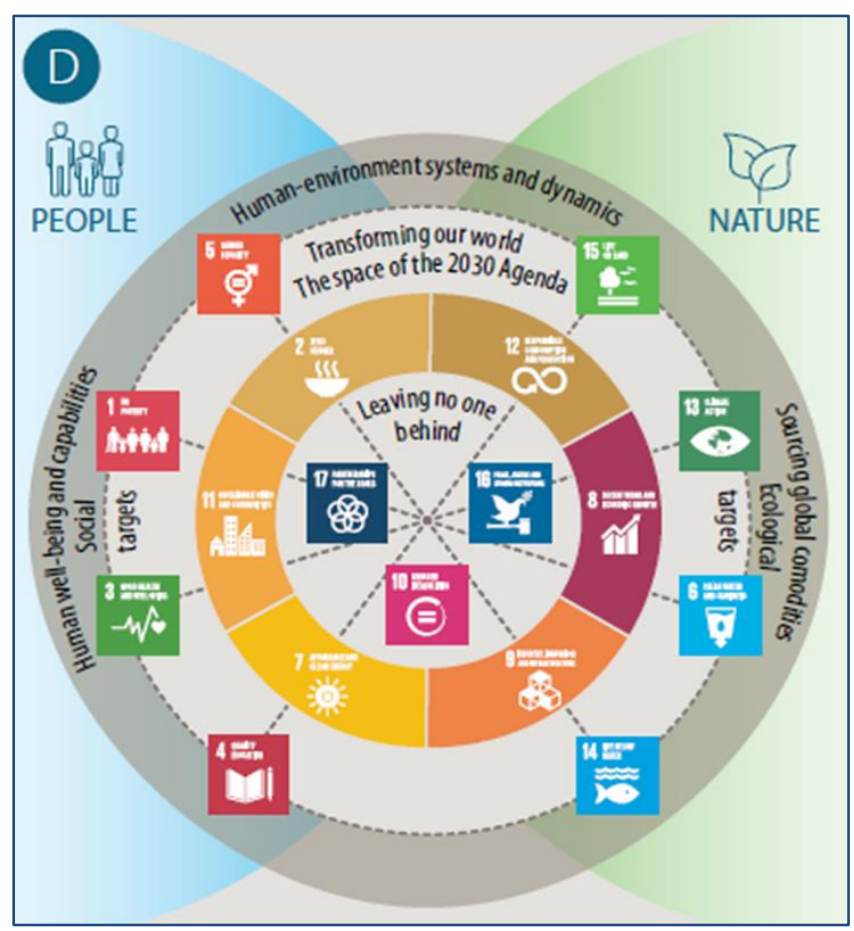

Figure 3. The knowledge-based transformations towards sustainable development (Source: TWI2050 - $\neg$ e World in $2050(2018)$

SDGs Today features real-time and timely data on the Sustainable Development Goals (SDGs) and offers educational resources to support countries, institutions, and civil society members to produce, share and engage with the data to help ensure that we meet the global goals by 2030 .

\section{Possible SCENARIOS}

Countries have not been able to reconcile significant tradeoffs in the implementation of development goals. For example, the linkages between the goals of human development, economic growth, and equality show catalytic effects on each other when policies to support these goals are implemented simultaneously, and the trade-offs across these different dimensions of progress should be interrogated further.
We argue that while greater well-being will require more economic diversification as well as more and better public services, it will also mean addressing the negative impacts of economic progress, such as environmental degradation and climate change to sustain win-win outcomes and avoid the risk of reversing improvements on the poorest people's lives. Very little is known about countries that have successfully navigated the different dimensions of tradeoffs. Others identify the causal paths of single elements of success and have not examined the possible trade-offs that were necessary to achieve this. We are facing some hard questions that have not been tackled to date through segmented approaches:

- How can ending hunger be reconciled with environmental sustainability? (SDG targets 2.3 and 15.2) - How can economic growth be reconciled with environmental sustainability? (SDG targets 9.2 and 9.4)

- How can income inequality be reconciled with economic growth? (SDG targets 10.1 and 8.1).

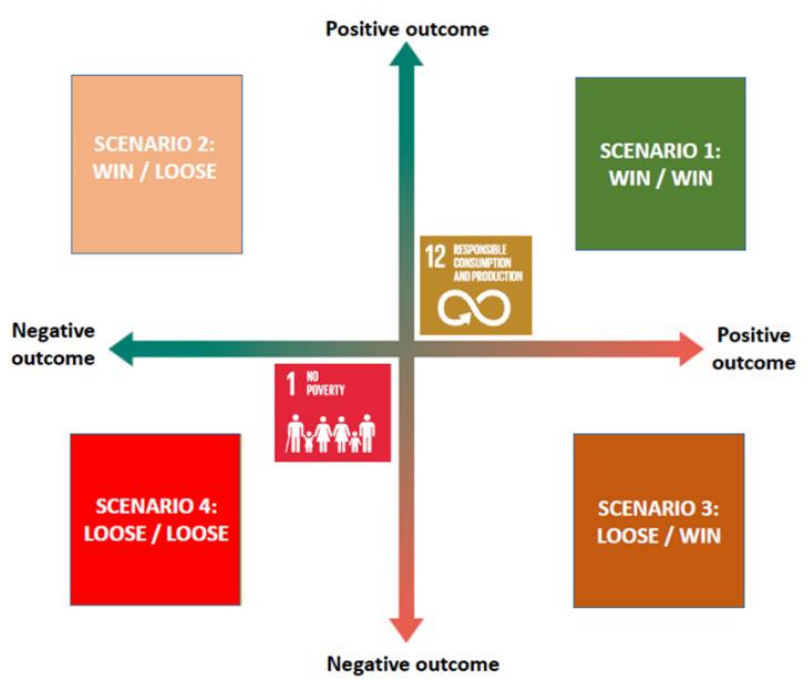

Figure 4. Possible future scenarios based on the success of complexity management - using examples of two SDGs (authors editing)

\section{Conclusions}

Development theory has tried to consider such types of questions like above and the implications of trade-offs but has often concluded that 'we don't know' or 'further research is needed' or 'we don't have the data'. For policymakers, however, these are not theoretical questions: they are part of everyday reality. The possibility that goals and their strong trade-offs could cancel each other out is - and will increasingly become - a major issue for SDG implementtation.

Tensions in scenario-building are often attributed to a onesided focus on either a structured approach, focused only on systems-thinking (plotting) but poor narrative-building 
(stories), or focused only on narrative-building (stories) but poor systems-thinking.

Tensions between scientific soundness and imagination are also possible weaknesses of scenario constructions. Normative scenario exercises that try to counter these weaknesses adopt structured approaches that pay attention to quantitative modeling but have also been criticized for failing to build a compelling scenario because of poor balances between the structured analysis and storyline approach.

We try to combine systems-thinking with narrative-thinking, and participatory with expert processes. We also try to balance a structured approach with an intuitive approach, remaining largely qualitative rather than quantitative.

The qualitative approach offered interpretive flexibility by integrating the disparate ideas, views, and feelings of expert stakeholders into one holistic picture while reflecting uncertainties.

\section{REFERENCES}

United Nations. IAEG-SDGs Tier Classification for Global SDG Indicators. 2019a.

Nilsson, M., Chisholm, E., Griggs, D. et al. Mapping interactions between the sustainable development goals: lessons learned and ways forward. Sustain Sci 13, 1489-1503 (2018).

DOI: $\underline{10.1007 / \mathrm{s} 11625-018-0604-\mathrm{Z}}$

United Nations. The Future is Now: Science for Achieving Sustainable Development - Global Sustainable Development Report: Department of Economic and Social Affairs, New York. 2019b.

https://sustainabledevelopment.un.org/content/documents/24 797GSDR_report_2019.pdf

TWI2050 e-World in 2050 (2018). Transformations to Achieve the Sustainable Development Goals. Report prepared by $\neg$ e World in 2050 initiative. International Institute for Applied Systems Analysis (IIASA), Laxenburg, Austria.

https://iiasa.ac.at/web/home/research/twi/Report2018.html

Gennari, P. and - Navarro, D. K. (2020): Are We Serious About Achieving the SDGs? A Statistician's Perspective. http://sdg.iisd.org/commentary/guest-articles/are-weserious-about-achieving-the-sdgs-a-statisticians-perspective/

PARIS21. 2020 Board Document - PARIS 21 Annual Meetings, 07-08 April 2020. pp 1 - 124.

https://paris21.org/sites/default/files/inline-

files/2020_BOARD_DOCUMENT_PARIS21_FINAL_1.pdf

(C) 2020 by the author(s). This article is an open-access article distributed under the terms and conditions of the Creative Commons Attribution (CC BY) license (http://creativecommons.org/licenses/by/4.0/). 